\title{
Anomalous Flow Behavior in Nanochannels: A Molecular Dynamics
}

\section{Study}

Sohail Murad ${ }^{\mathrm{a}, \mathrm{b}}$, Lin Luo ${ }^{\mathrm{c}(1)}$, and Liang-Yin Chu ${ }^{\mathrm{b}}$

${ }^{a}$ Department of Chemical Engineering University of Illinois at Chicago, Chicago, Illinois 60607, USA.

${ }^{\mathrm{b}}$ School of Chemical Engineering and ${ }^{\mathrm{c}}$ State Key Laboratory of Hydraulics, Sichuan University, Chengdu, Sichuan 610065, China

\begin{abstract}
We report molecular dynamics simulations of flow of water in nanochannels with a range of surface wettability characteristics (hydrophobic to strongly hydrophilic) and driving forces (pressures). Our results show apparently anomalous behavior. At low pressures, the rate is higher in nanochannels with hydrophilic surfaces than that with hydrophobic surfaces; however, with high pressure driven flow we observe opposite trends. This apparently anomalous behavior can be explained on the basis of molecular thermodynamics and fluid mechanics considerations. Understanding such behavior is important in many nanofluidic devices such as nanoreactors, nanosensors, and nanochips that are increasingly being designed and used.

\section{Introduction}

Nanochannels are passages with nano-scale dimensions through which fluids may move or be directed. They can be designed and used in myriads of applications, including lab-on-a-chip systems $^{1-6}$, nanofluidics ${ }^{7-9}$, and porous membranes ${ }^{10-13}$. Rational manipulation of fluid flow in

\footnotetext{
${ }^{1}$ This paper is dedicated to Prof. Lin Luo, who died suddenly on October 11, 2009 while this work was in progress.
} 
nanochannels is of great technological importance for designing better, smaller, and cheaper devices. Here we report flow behaviors in nanochannels with changing surface wettability using the method of molecular dynamics. Amongst other observations, our results show that at low pressures, the water flux through nanochannels with hydrophilic surface is larger than that through nanochannels with the same dimension but with hydrophobic surface. However, surprisingly the situation is reversed under high pressure.

Nanofluidics, microfluidics, and membranes have the potential to influence diverse fields including chemical synthesis/separation and biological analysis/separation to optics and information technology ${ }^{1}$. The manipulation of fluids in nanochannels and microchannels has attracted much attention from physicists and chemists ${ }^{14}$, biologists, as well as engineers. The wetting behavior of a solid surface by a liquid is a very important aspect of surface chemistry, and the surface wettability of a channel or passage has been found to be crucial for effective fluid flow $^{15}$. However, due to the lack of suitable instruments, it is still too hard to carry out experiments to investigate the flow behaviors of fluid in nanochannels in details. Fortunately, molecular dynamics simulations appear to be a useful potential tool to study the fluid flow behaviors in nano-scale channels, which has been used in this study.

\section{Method and Models}

To probe into the flow phenomena in nanochannels, molecular dynamics simulations are performed for water flowing through nanochannels with changing surface wettability. The initial setup of our simulation system is shown in Figure 1. It consists of a parallelepiped with fixed and moving walls. Wall molecules are shown in blue, and moving walls have a white outline, with an arrow to show the direction in which they move during the simulation. Water is shown as pink molecules in the simulation box. The walls are kept stationery in free flow, while they 
are moved down at a fixed rate for forced flow. The molecules that constitute the walls/surface have no charges for hydrophobic walls, while in the case of hydrophilic walls the charges on the wall molecules are 0.33e (in reduced units $Q=3.5$ ) in our "standard" hydrophilic case, with an equal number of positive and negative charges to make the wall surface net neutral. We have carried out a range of studies in which this charge is varied to make the walls more hydrophilic (larger charges) or less hydrophilic (smaller charges). The usual lengths of the simulations consist of 2 million time steps, with each step approx. 0.16 femtosecond. The system consists of 6144 particles and is approximately $12 \mathrm{~nm}$ long ( $x$ direction), while the channel is $6 \mathrm{~nm}$ long and the width is $1 \mathrm{~nm}$. Water is modeled in our simulations using the simple point charge (SPC) potential ${ }^{16}$, while the nanochannel atoms are modeled either as Lennard-Jones (LJ) sites to model hydrophobic walls, or as LJ sites with alternating positive and negative charges to model hydrophilic walls ${ }^{17}$. The simulations are carried out with water at $334 \mathrm{~K}$. The walls are modeled by tethering the atoms that constitute the nanochannel using a simple harmonic potential. It is these tethering sites that are selectively moved (see Figure 1) to force the walls to get dragged along, while allowing for thermal fluctuations. In a subset of the simulations, we replace the top surface layer of the nanochannels with tethered water molecules (see Figure 2, tethered water shown in yellow), as an alternate scheme for modeling hydrophilic nanochannel surfaces. We will refer to this as type II hydrophilic channel surface and the former with alternating charges as type I. The MD simulation algorithm uses the quaternion method ${ }^{18}$ with a fifth-order Gear predictor-corrector algorithm for translational motion and a fourth-order predictor-corrector algorithm for rotational motion. The "standard" compression rate in our forced flow studies was $1.08 \mathrm{~m} / \mathrm{s}$, but several studies with a range of compression rates were also carried out. 


\section{Results and Discussion}

The results obtained from our simulation for water flow/penetration in the nanochannels are shown in Figures 2 and 3 for free flow (spontaneous wetting driven) and forced flow (standard compression rate, $1.08 \mathrm{~m} / \mathrm{s}$ ) for both hydrophobic and hydrophilic surfaces (type I and II). Figures 2(a) and 2(b) compare results for free flow for hydrophobic and type I hydrophilic surfaces. In the case of free flow, water is not forced to enter the nanochannels by compressive forces, so flow is essentially driven by energetic (wetting) considerations. For the hydrophobic case we observe no flow at all, because water molecules would prefer minimizing contact with the wall surface, as that would increase their internal energy. For the hydrophilic case, water molecules lower their energy by entering the nanochannels and this flow is driven by the nanoscale equivalent of capillary action flow. The relative interactions between water/water and the water/surface, would determine the flow rate in the nanochannels. In our case since a finite amount of water is available for flow in the channel (see Figure 2), the channel may not be completely filled at equilibrium (our simulations were not long enough to reach equilibrium). However, if there were an infinite supply of water, the flow would reach a steady state value. Figures 2(c) and 2(d) show results for hydrophobic and hydrophilic surfaces (red sites) with a top monolayer of tethered water (yellow) in the channels. Our results show that despite the fact the channel surface is identical in both cases, the flow rates in the channels are different. This is caused by the behavior of the surface in the reservoir (left side) which in the case of hydrophobic surfaces tends to move the water into the channel because of more favorable energetics (water would have lower energy in contact with water on the surface of the channel than the reservoir). This leads to higher flow rates (defined here as the linear flow rate in the nanochannel in meters per second, m/s) in the hydrophobic reservoir case. In addition, the flowrate in Figure 2(d) is 
lower than that in Figure 2(b). This is because the surface in Figure 2(b) is more hydrophilic than that in Figure 2(d). For the results reported here, steady behavior is observed after a short transition period in the forced flow cases (due to the constant compression rate) till the reservoir is almost empty. For the free flow cases steady behavior also results, till surface forces causes cavities to develop in the liquid region, when the flow rates tend to slow down. The flow rates reported here are from the steady flow regions.

For the case of forced flow, as is clear from the results in Figures 3(a) and 3(b), the flowrate is higher for the hydrophobic case. These results can be understood if one examines the relative strength of the intermolecular forces between two water molecules and the water molecules and the surface. When water is compressed in the reservoir it is forced to enter the nanochannel. Once inside the nanochannels, in the case of hydrophilic surfaces the interactions between the water molecules and the surface are stronger than those between two water molecules (this was also clearly shown when comparing Figures 2a and 2d). The water molecules therefore tend to maximize their contact with the surface and the water molecules are also in closer contact with the surface, as can also be seen in the Figures 2 and 3. This results in making the movement of water molecules adjacent to these molecules more difficult because of drag resistance. This also leads to the concave surface observed. For hydrophobic surfaces the interactions between two water molecules are stronger than those between water and the channel surface. Thus water molecules tend to minimize their contact with the surface and the water in the channel tends to be further away from the surface compared to the hydrophilic case. Water being forced into the nanochannels thus can flow via plug flow ${ }^{19}$ with relatively little drag resistance. In the cases where a layer of water is tethered the results are similar, but since in this case the surface as stated earlier is less hydrophilic, the differences between the two cases are 
less pronounced.

We can also examine the effect of surface characteristic in the flow resistance by varying the rate at which the fluid is compressed. In Figure 4, we show the results for both hydrophobic and a range of hydrophilic surfaces (type I), ranging from weakly hydrophilic to strongly hydrophilic (stronger than surfaces covered by a layer of water itself, see Figures 2-3) as a function of compression rate. It can be seen clearly that for hydrophobic surfaces, the flow rate is linearly related to the compression rate, which a fluid mechanics analysis would point to frictionless flow ${ }^{19}$ (if the flow were frictional, then the frictional force would have increased with the flow rate, and the linear behavior observed would not have been possible), but in the case of hydrophilic surfaces is compression rate dependent, which points to flow resistance changing as a function of changes in flow rate. Figure 4 also shows that the effect of the magnitude of the hydrophilicity (defined as the magnitude of similarly distributed charges on the sites) is clearly not linear. The behavior of the weakly hydrophilic surface is indistinguishable from the hydrophobic, except for a small difference for free flow. For moderately hydrophilic surface we observe transition behavior. At low compression rates the behavior is linear similar to hydrophobic surfaces (except for some free flow), but at higher compression rates nonlinear behavior is observed as in hydrophilic surfaces.

The effect of surface wettability characteristics on the flow rate under compression has been examined further in Figure 5 with our standard compression rate $(1.08 \mathrm{~m} / \mathrm{s})$. It can be seen when the surface is only slightly hydrophilic ( $Q=1.0$, which corresponds to charges of $0.11 \mathrm{e}$ ) the differences between the hydrophilic and hydrophobic cases is minimal. At $Q=2.0$, differences become noticeable, but by $Q=5.0$, it is almost impossible for the fluid to flow. We would like to point out that since the reservoir boundary molecules are tethered, when the pressure inside 
becomes excessive, some molecules leak out, as they perhaps would with a safety valve, to prevent the reservoir from exploding. We also looked at the behavior as a function of surface characteristics (hydrophobic $\rightarrow$ hydrophilic) at half and 1.5 times this compression rate. The differences become less pronounced at the lower compression rate and more pronounced at the higher compression rates. However for the case $Q=1.0$, there continue to be almost no differences in the flow rate even at the higher compression rates, pointing to almost frictionless flow here as well.

It is also possible to compare the wicking distance obtained from molecular dynamics simulations with continuum hydrodynamics using Young-Laplace equation ${ }^{20}$ with zero contact angle, if it is assumed that the capillary pressure jump across interface is providing the driving force for flow. If fully developed rectilinear viscous flow neglecting rearrangement at the channel entrance and meniscus are assumed, one obtains the equation

$$
\frac{d l}{d t}=\left(\frac{\gamma}{6 \mu H}\right) \frac{1}{l}
$$

Where $l$ is the wicking distance, $t$ the time, $\mu$ the viscosity, $H$ the channel width, and $\gamma$ the surface tension. If our simulation results are compared with the above equation, for a wicking distance of $3 H$, away from the entrance region, the molecular dynamics simulations results give a time of approximately $2 \mathrm{E}-10$ seconds, while the continuum results are $4 \mathrm{E}-10$; considering the assumptions made, an extraordinary agreement.

\section{Conclusions}

In conclusion, our studies have shown rather anomalous behavior of water flow in nanochannels. For hydrophobic surfaces the flow of water appears to be almost frictionless Knudsen flow ${ }^{20}$, but there is no wetting (free flow). For hydrophilic surfaces, while significant capillary (wetting flow) is observed, the surface strongly resists rapid movement of water 
molecules, because of strong water-surface interactions. This also leads to high pressures in the compression chamber in Figure 1 as evidenced by the leakage observed from the reservoir walls. In nanochannels the wettability characteristics of the surface have a profound effect on the flow behavior, and as nanofluidic devices become more prevalent, these effects will increasingly have to be accounted for when designing such nanofluidic devices. Molecular dynamics simulations appear to be a very useful tool to study such phenomena in nano-scale dimensions.

Acknowledgments. We would like to thank Prof. L.C. Nitsche for many helpful discussions. This research has been funded by a grant from the Office of Basic Energy Science, Department of Energy [Grant No. DE-FG02-08ER46538] and a grant from the National Natural Science Foundation of China [Grant No. 20825622]. Travel funds were provided by the National Science Foundation (CBET 0314203)

\section{References}

1. Whitesides, G. M. The origins and the future of microfluidics. Nature 442, 368-373 (2006).

2. deMello, A. J. Control and detection of chemical reactions in microfluidic systems. Nature 442, 394-402 (2006).

3. Fuerstman, M. J., Garstecki, P. \& Whitesides, G. M. Coding/decoding and reversibility of droplet trains in microfluidic networks. Science 315, 828-832 (2007).

4. Utada, A. S., Lorenceau, E., Link, D. R., Kaplan, P. D., Stone, H. A. \& Weitz, D. A. Monodisperse double emulsions generated from a microcapillary device, Science 308, 537541 (2005).

5. Atencia, J. \& Beebe, D. J. Controlled microfluidic interfaces. Nature 437, 648-655 (2005). 
6. Thorsen, T., Maerkl, S. J. \& Quake, S. R. Microfluidic large-scale integration. Science 298, 580-584 (2002).

7. Han, J. \& Craighead, H. G. Separation of long DNA molecules in a microfabricated entropic trap array. Science 288, 1026-1029 (2000).

8. Fu, J., Schoch, R. B., Stevens, A. L., Tannenbaum, S. R. \& Han, J. A patterned anisotropic nanofluidic sieving structure for continuous-flow separation of DNA and proteins. Nature Nanotech. 2, 121-128 (2007).

9. Huh, D., Mills, K. L., Zhu, X., Burns, M. A., Thouless, M. D. \& Takayama, S. Tuneable elastomeric nanochannels for nanofluidic manipulation. Nature Mater. 6, 424-428 (2007).

10. Striemer, C. C., Gaborski, T. R., McGrath, J. L. \& Fauchet, P. M. Charge- and size-based separation of macromolecules using ultrathin silicon membranes. Nature 445, 749-753 (2007).

11. Savariar, E. N., Krishnamoorthy, K. \& Thayumanavan, S. Molecular discrimination inside polymer nanotubules. Nature Nanotech. 3, 112-117 (2008).

12. Baker, L. A. \& Bird, S. P. A makeover for membranes. Nature Nanotech. 3, 73-74 (2008).

13. Yuan, J., Liu, X., Akbulut, O., Hu, J., Suib, S. L., Kong, J. \& Stellacci, F. Superwetting nanowire membranes for selective absorption. Nature Nanotech. 3, 332-336 (2008).

14. Whitby, M. \& Quirke, N. Fluid flow in carbon nanotubes and nanopipes. Nature Nanotech. 2, 87-94 (2007).

15. Choi, C. H., Westin, K. J. A. \& Breuer, K. S. Apparent slip flows in hydrophilic and hydrophobic microchannels. Phys. Fluids 15, 2897-2902 (2003).

16. Berendson, H. J. C., Postma, J. \& Gunsteren, W. F. in: Pullman, B. (ed.), Intermolecular Forces (Reidel, Dordecht, 1981). 
17. Murad, S. \& Puri, I. K. Nanoscale jet collision and mixing dynamics. Nano Letters 7, 707712 (2007).

18. Evans, D. J. \& Murad, S. Singularity free algorithm for molecular dynamics simulation of rigid polyatomics, Molecular Physics 34, 327-331 (1977).

19. Bird, R.B., Stewart, W.E., \& Lightfoot, E.N. Transport Phenomena ( ${ }^{\text {nd }}$ Edn) (WileyInterscience, NY, 2002).

20. Adamson, A. W. \& Gast, A. P. Physical Chemistry of Surfaces ( $6^{\text {th }}$ Edn) (Wiley-Interscience, NY, 1997). 


\section{Figures}

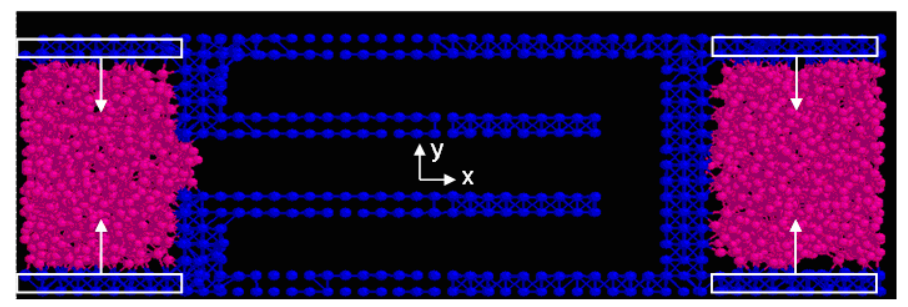

Figure 1. A schematic of the system setup. The two walls on each side with white outlines move to compress the fluid resulting in forced flow. In the case of capillary flow, the fluid is not compressed. 


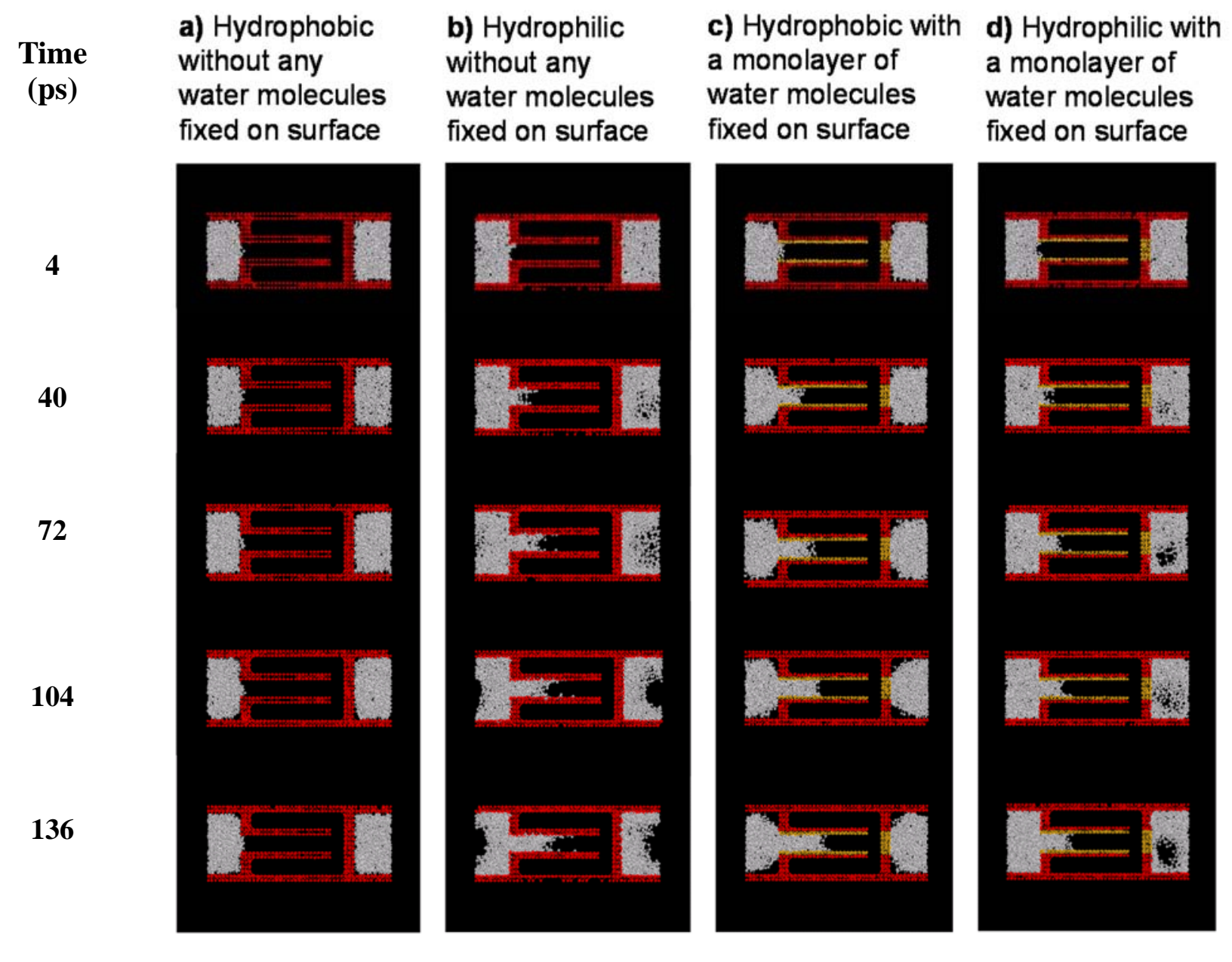

Figure 2. Capillary flow of water in nanochannels with hydrophobic (a) and hydrophilic (b) wall surfaces. To verify the effect of surface wettability on the water flow behavior, a monolayer of water molecules is fixed on both hydrophobic (c) and hydrophilic (d) surfaces for comparison. 


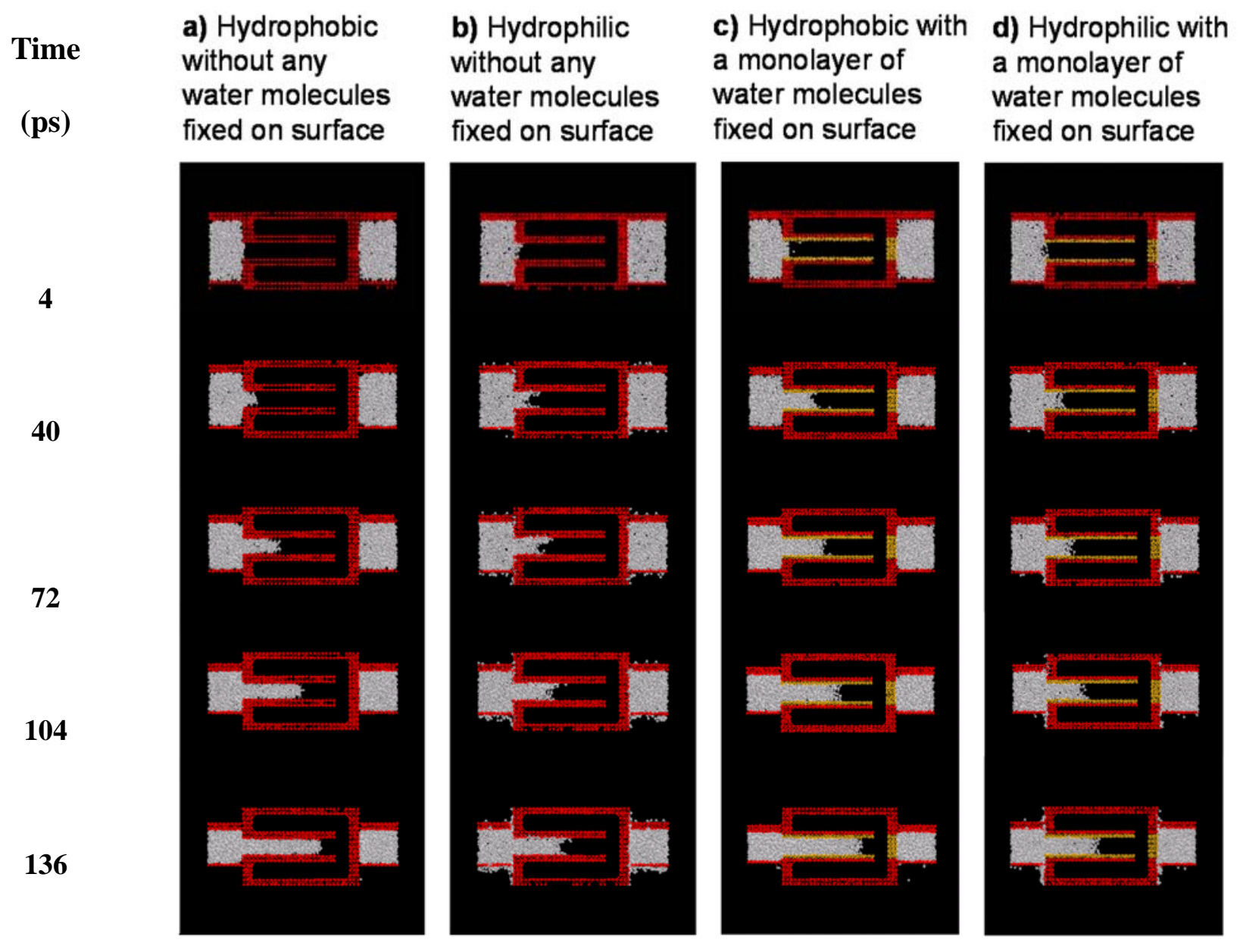

Figure 3. Forced flow of water in nanochannels with hydrophobic (a) and hydrophilic (b) wall surfaces. To verify the effect of surface wettability on the water flow behavior, a monolayer of water molecules is fixed on both hydrophobic (c) and hydrophilic (d) surfaces for comparison. The compression rate is $1.08 \mathrm{~m} / \mathrm{s}$. 


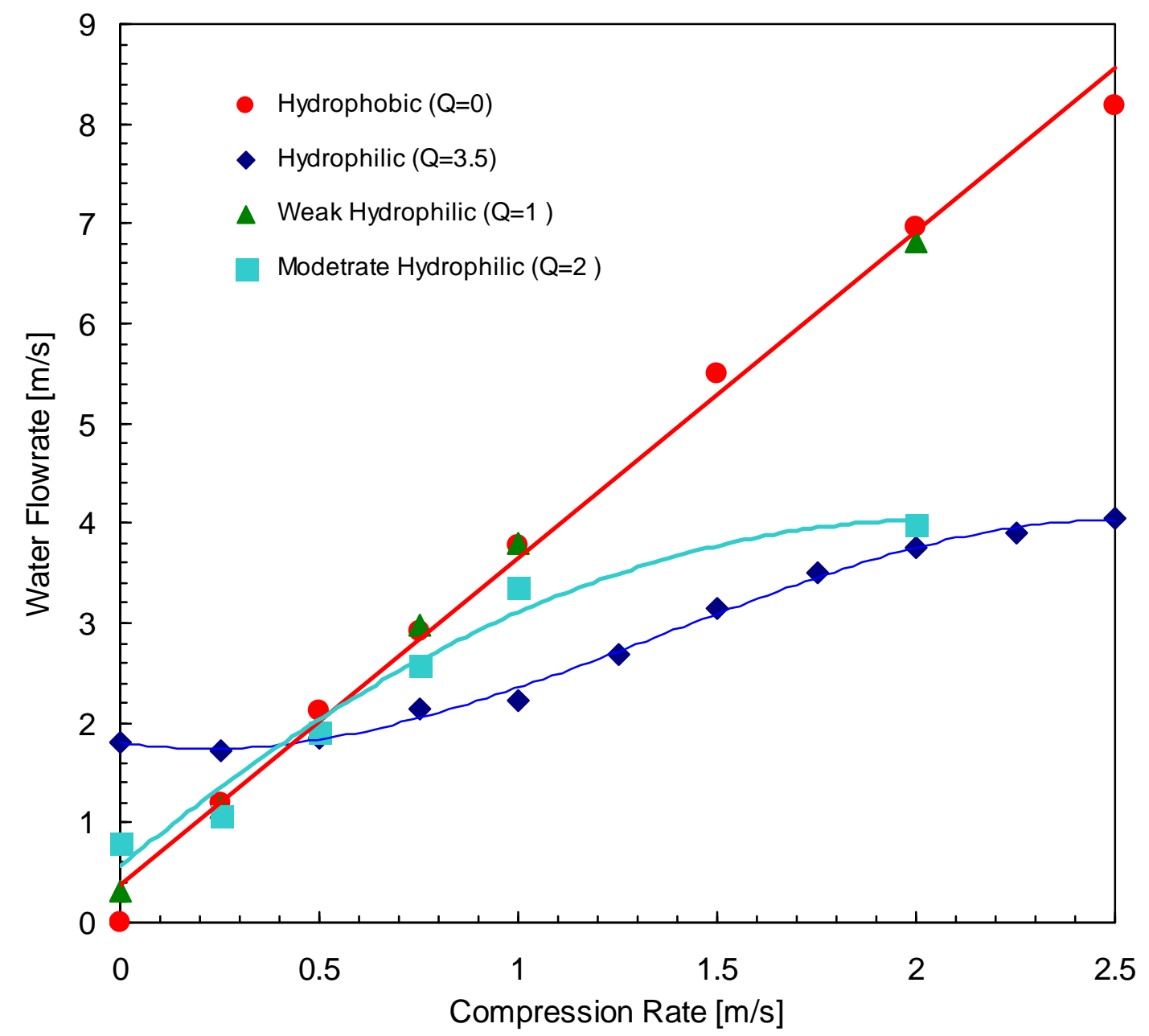

Figure 4. Flow velocity of water in nanochannels with hydrophobic and hydrophilic surfaces as a function of compression rate. 


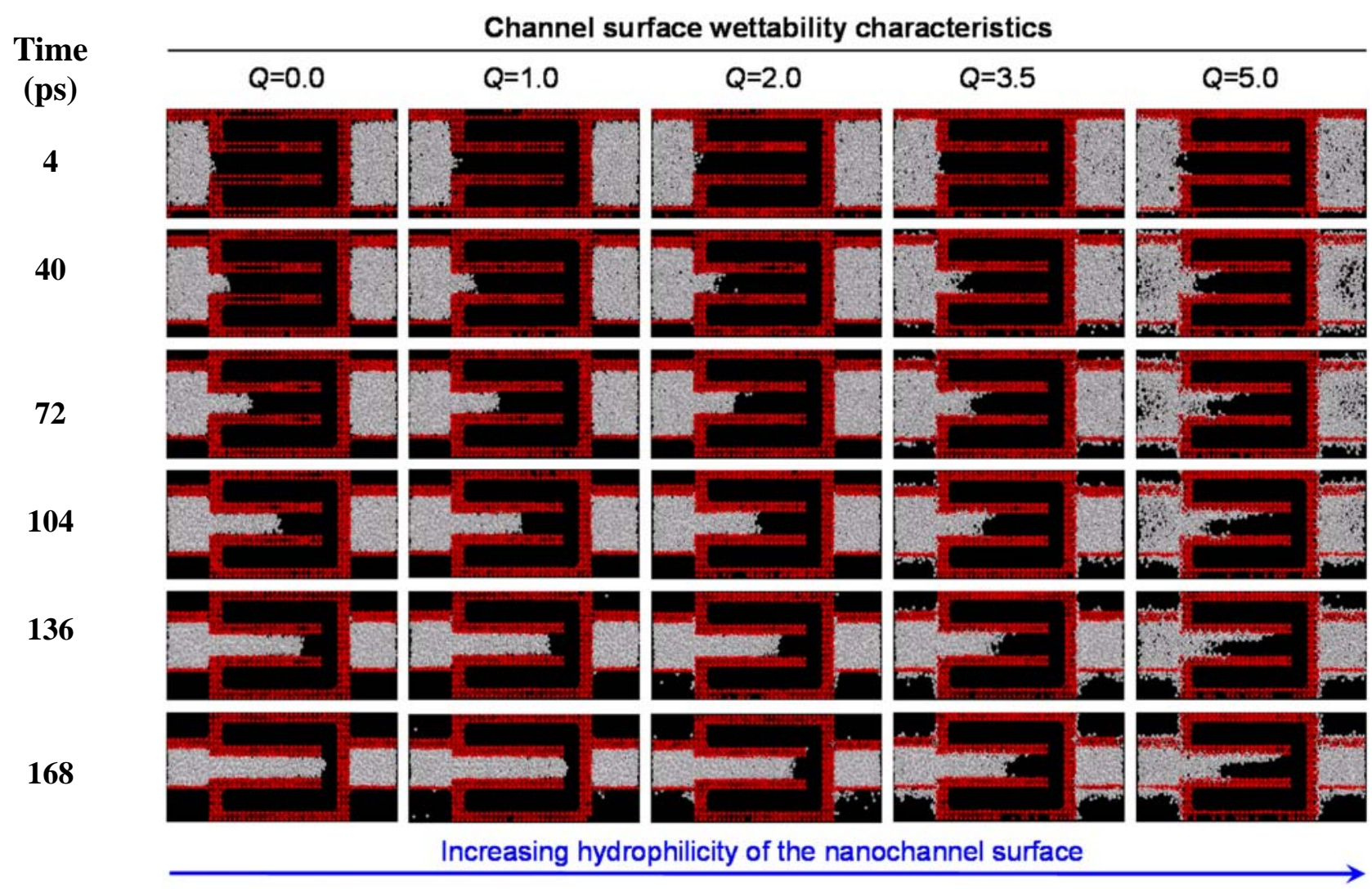

Figure 5. Effect of channel surface wettability on the forced flow rate of water in nanochannels. The compression rate is $1.08 \mathrm{~m} / \mathrm{s}$. 